\title{
A proposal for a level for parapharyngeal extension of parotid gland
}

\author{
Nicolas Fakhry ${ }^{1}$
}

Received: 27 June 2016/Accepted: 19 July 2016/Published online: 25 July 2016

(C) Springer-Verlag Berlin Heidelberg 2016

\section{Dear Editor,}

We read with great interest the recently published article by Quer et al. [1]. "Classification of parotidectomies: a proposal of the European Salivary Gland Society" that aimed to propose a classification system to have a common way to report surgery and to facilitate scientific communication. They have divided parotid gland into five levels including a level $\mathrm{V}$ for anterior extension of parotid gland and then have defined surgical classification depending on tissue removal and surgical approach. This classification will be certainly very useful. It is our view that parotid parapharyngeal extension should be considered as a level due to specificity of surgery for tumors arising from this very deep part of parotid gland. Parapharyngeal space is divided into prestyloid and retrostyloid compartments by the stylopharyngeal fascia and the prestyloid space contains the extension of the deep lobe of parotid gland [2]. These anatomical considerations can lead to radically different surgical strategies depending on tumor histology (benign or malignant) and tumor extension into areas surrounding the prestyloid space including infra-temporal fossa and retrostyloid space [3-5]. While some benign tumors, located in the parapharyngeal space parotid extension, can be removed with a limited approach using,

This comment refers to the article available at doi:10.1007/s00405016-3916-6.

Nicolas Fakhry

nicolas.fakhry@ap-hm.fr

1 Service d'ORL et Chirurgie Cervico-Faciale, Assistance Publique-Hôpitaux de Marseille (AP-HM), Centre Hospitalier Universitaire (CHU) La Conception, Aix Marseille Univ, 147 Boulevard Baille, 13005 Marseille, France for example, partial inferior parotidectomy to access the tumor, in other cases the approach require a more aggressive surgery with, for example, a trans-mandibular approach.

We believe that add a level for parapharyngeal extension of parotid gland would be useful in the classification.

\section{Compliance with ethical standards}

Conflict of interest None.

\section{References}

1. Quer M, Guntinas-Lichius O, Marchal F et al (2016) Classification of parotidectomies: a proposal of the European Salivary Gland Society. Eur Arch Otorhinolaryngol. doi:10.1007/s00405-0163916-6

2. Varoquaux A, Fakhry N, Gabriel S et al (2013) Retrostyloid parapharyngeal space tumors: a clinician and imaging perspective. Eur J Radiol 82:773-782

3. Eisele DW, Richmon JD (2013) Contemporary evaluation and management of parapharyngeal space neoplasms. J Laryngol Otol 127:550-555

4. Bradley PJ, Bradley PT, Olsen KD (2011) Update on the management of parapharyngeal tumours. Curr Opin Otolaryngol Head Neck Surg 19:92-98

5. Kuet ML, Kasbekar AV, Masterson L, Jani P (2014) Management of tumors arising from the Parapharyngeal space: a systematic review of 1293 cases reported over 25 years. Laryngoscope. doi:10.1002/lary.25077 